\title{
Analysis of Psychological Contract Effectiveness in Business Process Transformation
}

\author{
Kayo Iizuka and Chihiro Suematsu
}

\begin{abstract}
Business process transformation (BPT) is one of the most useful methods for companies or other types of organizations to survive in the turbulent business environment. Numbers of studies have contributed to improving the effectiveness of BPT from the perspectives of such as project management of BPT, and analysis of the critical success factors of BPT. As for cases in Japan, there are some unique issues relevant to changing overall business processes, including operations of the back offices which throughout all industries are not always efficient [1.2]. Therefore, in addition to the BPT methodologies used in Western countries, it is necessary to seek for the counterpart for the unique issues of BPT in Japan. In this paper, the authors focus on the correlation between psychological contracts and BPT effect as one of the success factors for BPT.
\end{abstract}

Index Terms-Business process transformation (BPT), psychological contract, success factor.

\section{INTRODUCTION}

The word "KAIZEN (continuous improvement)" has become popular all over the world. It has been said for some decades that overall quality and efficiency of production lines in Japan are superior to those in other countries. However, overall business processes, especially operation in the back offices are not always efficient [1], [2], and labor productivity in Japan ranks 22nd out of 34 countries according to the OECD [3]. Companies in Japan are trying to make their business processes more efficient by using Information Technology (IT). Business process transformation (BPT) is one of the most useful methods for companies or other types of organizations to sustain themselves as they adapt to business environmental changes. Higano mentioned that Japanese companies tend to spend much more, in percentage terms, on improving operational efficiency of their businesses compared to companies in Western countries, but the results do not seem to be satisfactory because the contribution of IT investment to value added growth in Japan is lower than that in Western countries [4]. There are many methodologies for BPT such as business process re-engineering (BPR) and business process integration (BPI) [5]. However, it is difficult to

Manuscript received March 13, 2019; revised May 23, 2019. This work was supported in part by Grant-in-Aid for Scientific Research (16K03819).

K. Iizuka is with the Senshu Unversity, Kawasaki, Kanagawa, Japan (e-mail: iizuka@isc.senshu-ac.jp).

C. Suematsu is with the Graduate School of Management, Kyoto University, Kyoto, Japan (e-mail: suematsu@econ.kyoto.ac.jp). achieve the objectives only by applying ideal or picture-perfect models. Thus, the authors focused on trust in the organizations; trust between managers and employees, and trust between the different sections of companies. To do as such, the authors focus on the correlations between the psychological contracts and BPT effect as one of the success factors for BPT from a perspective of trust.

\section{RELATED WORK}

The concept of psychological contract was developed by Rousseau. It was defined as employees' beliefs concerning their exchange agreement with their employer and the organization [6], [7]. Psychological contract develops within a dynamic environment in which the individual is often interacting with multiple organizational agents who may be sending messages each other, on both verbal and non-verbal manner [8]. It is impossible for employees to have official contracts with their employer or the organization for every possible cases. Therefore, considering the influence of psychological contracts is important for managing organizations, not only for managing ordinary working organizations, but for ad hoc organizations such occurs in projects. The use of project management (PM) technology is a competence that organizations must develop, understanding that PM is the capability to attend to the results that are needed to maintain organizations' sustainability, aligning the mission and vision and optimizing the resource allocation to maintain its competitive advantages [9]. BPT is one of the most important project activities for companies or other types of organizations, and is conducted in order to realize change in their business processes and IT to survive in the business environment that is rapidly changing. Numbers of studies have contributed to improving the effectiveness of BPT, for example through business process re-engineering (BPR) and business process modeling. Hammer and Champy defined BPR as a fundamental rethinking and radical redesign of business processes to achieve dramatic improvements in critical, and contemporary measures of performance, such as cost, quality, service and speed [10]. Grover et al. focused on the implementation problem [11]. Earl et al. analyzed the relationship between BPR and strategic planning [12]. Attaran explored the relationship between IT and BPR based on the capabilities and barriers to effective implementation [13]. There are many studies dealing with BPR success and failure factors [14], [15]. However, the success factors of BPT sometimes vary from country to country [16], and, it is significant to seek for the counterpart 
of methodologies used in Western countries, taking the consideration for the unique issues of BPT in Japan. Therefore, to focus on the correlation between psychological contract and BPT effect as one of the success factors for BPT would be relevant to improving BPT effectiveness.

\section{RESEARCH MODEL}

The purpose of this study is to clarify the correlation between the psychological contracts and the BPT effect. The research model is shown in Fig. 1.

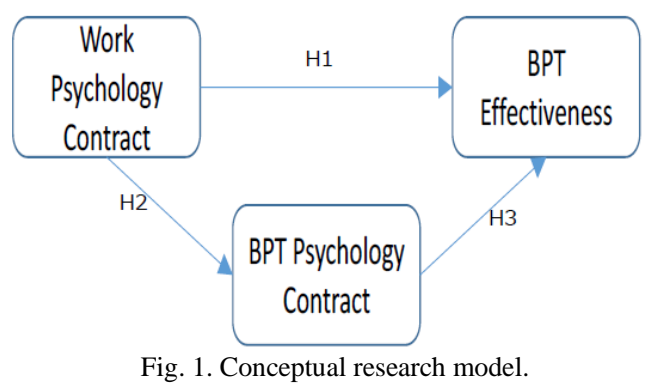

The hypotheses created for this survey are as follows:

H1: BPT psychology contract has positive impact on BPT effectiveness.

Since BPT effect is considered to be related to trust, and the psychological contract seems to be an important factor in forming trust, we formed the hypothesis that the BPT psychology contract has a positive impact on BPT effectiveness.

H2: Psychology contract of ordinary work environment (Work Psychology Contract) has positive impact on BPT psychology contract.

Since the BPT psychological contracts are considered to have a relationship with the psychological contracts of everyday working, the authors formed this hypothesis.

H3: Work psychology contract has a positive impact on BPT effectiveness.

Hypotheses 1 and 2 are concerned with the indirect relationship between work psychological contracts and BPT effect. However, it can be considered that there will be a direct relationship between work psychological contracts and BPT effect.

The authors planned to use the internet survey system to conduct a survey on who had engaged in BPT projects in a company or organization. Some of the survey questions are listed as follows:

(Psychology contract of usual working environment) [Work_Phy_Con]

1) I believe that my employer pays me an appropriate amount of salary deserves my job. [Q5S1]

2) I believe that my employer gives me appropriate performance feedback for my work. [Q5S2]

3) I believe that my employer assigns me suitable jobs for me. [Q5S3]

4) I believe that my employer supports me for my job when necessary. [Q5S4]

5) I believe I can engage in work that I feel it is intriguing. [Q5S5]
6) I believe that there are appropriate career options for me in my company. [Q5S6]

7) I believe that I can improve my skills and knowledge through my job. [Q5S7]

8) I believe that I can develop human networks through my job. [Q5S8]

(Psychology contract of BPT) [BPT_Psy_Con]

9) I believe that I can accomplish BPT effectively. [Q7S1]

10) I believe that I can obtain feedbacks regarding the total BPT effect on the company. [Q7S2]

11) I believe that my division can gain feedback about the total BPT effect on the company. [Q7S3]

12) I believe that I can obtain feedback regarding to my contribution. [Q7S4]

13) I think that the BPT support system is well arranged in my company. [Q7S5] (BPT effect) [BPT_Effect]

14) The BPT projects had succeeded in my company. [Q10S1]

Question items regarding psychological contracts of ordinary working environment are determined by reference to the studies of the psychological contract for the usual working environment [17], [18]. Question items of BPT psychological contracts and BPT effect are determined regarding the interview to the people who has experience about BPT.

\section{RESEARCH RESULT}

\section{A. Summary of Survey}

The data were collected in February 2019 through the internet survey system. The respondents were those who work for the companies or other types of organizations. There were 182 valid responses, and for the number of respondents who answered that they had experience of engaging more than one BPT project excluding on-going project were 32 .

\section{B. Analysis}

The analysis results based on our hypotheses are shown in Fig. 2 and Table I.

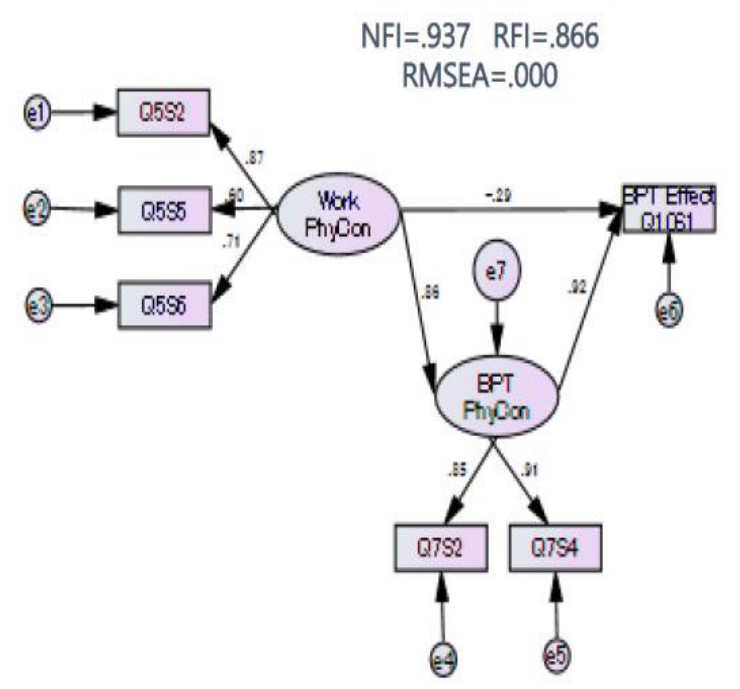

Fig. 2. The path coefficient map of psychological contract and BPT effect (1). 
TABLE I: REGRESSION WEIGHT AND STANDARDIZED REGRESSION OF BPT EFFECT (1)

Regression Weight

\begin{tabular}{lcccccc}
\hline \hline & & & $\begin{array}{c}\text { Estimat } \\
\text { e }\end{array}$ & S.E. & C.R. & P \\
\hline BPT_PsyCon & $<--$ & Work_PsyCon & .767 & .164 & 4.662 & $* * *$ \\
\hline Q5S2 & $<---$ & Work_PsyCon & 1.022 & .177 & 5.774 & $* * *$ \\
\hline Q556 & $<--$ & Work_PsyCon & .805 & .182 & 4.426 & $* * *$ \\
\hline Q5S5 & $<--$ & Work_PsyCon & .697 & .197 & 3.533 & $* * *$ \\
\hline BPT_Effect & $<--$ & Work PsyCon & -.295 & .464 & -.635 & .525 \\
\hline BPT_Effect & $<--$ & BPT PsyCon & 1.039 & .525 & 1.980 & .048 \\
\hline Q7Q2 & $<---$ & BPT PsyCon & 1.000 & & & \\
\hline Q7Q4 & $<---$ & BPT PsyCon & 1.179 & .184 & 6.418 & $* * *$ \\
\hline \hline Standardized Regrion Weight & & & & & \\
\hline
\end{tabular}

\begin{tabular}{lccc}
\hline \hline \multicolumn{3}{l}{ Standardized Regression } & \\
\hline BPT_PsyCon & $<\ldots-$ & Work_PsyCon & .863 \\
\hline Q5S2 & $<--$ & Work_PsyCon & .870 \\
\hline Q5S6 & $<--$ & Work_PsyCon & .712 \\
\hline Q5S5 & $<--$ & Work_PsyCon & .596 \\
\hline BPT_Effect & $<--$ & Work PsyCon & -.293 \\
\hline BPT_Effect & $<--$ & BPT PsyCon & .919 \\
\hline Q7Q2 & $<--$ & BPT PsyCon & .855 \\
\hline Q7Q4 & $<--$. & BPT PsyCon & .905 \\
\hline \hline
\end{tabular}

The direct correlation between work psychological contracts and the BPT effect was not significant. Thus, we tried to improve our model as in Fig. 3 and Table II.

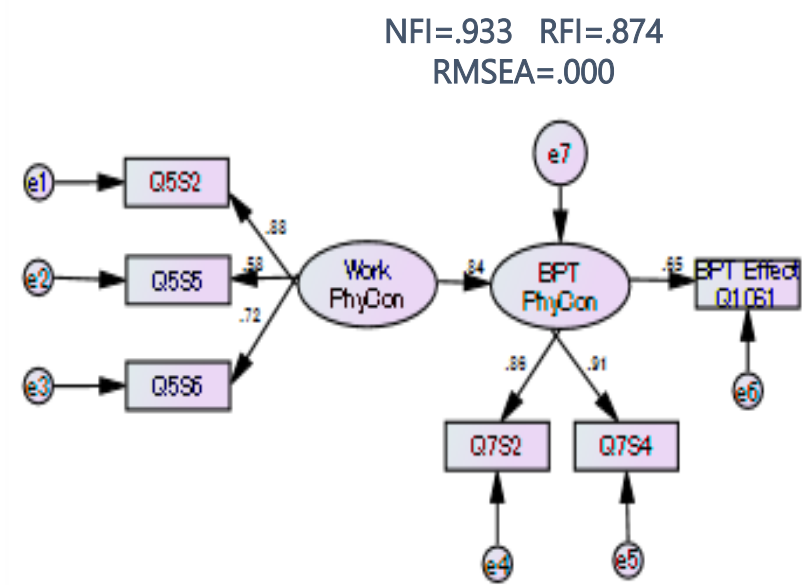

Fig. 3. The path coefficient map of psychological contract and BPT effect. (2).

The latent variable, the work psychological contracts formed by the observed variables, [Q5S2](I believe that my employer gives me appropriate performance feedback on my work), [Q5S5] ( I believe I can engage in work that I feel it is interesting) and [Q5S6] (I believe that there are appropriate career options for me in my company) has positive impacts on the latent variable, the BPT psychological contracts formed by the observed variables, [Q7S2] (I believe that I can gain feedback about the total BPT effect of the company)and [Q7S4](I believe that I can gain feedback corresponding to my contribution). A positive impact was seen between latent variable BPT psychological contract and BPT effect.

TABLE II: REGRESSION WEIGHT AND STANDARDIZED REGRESSION OF BPT EFFECT (2)

Regression Weight

\begin{tabular}{lcccccc}
\hline \hline & & & Estimate & S.E. & C.R. & P \\
\hline BPT_PsyCon & $<--$ & Work_PsyCon & .748 & .165 & 4.527 & $* * *$ \\
\hline Q5S2 & $<---$ & Work_PsyCon & 1.037 & .176 & 5.885 & $* * *$ \\
\hline Q5S6 & $<---$ & Work_PsyCon & .810 & .182 & 4.463 & $* * *$ \\
\hline Q5S5 & $<---$ & Work_PsyCon & .675 & .198 & 3.407 & $* * *$ \\
\hline BPT_Effect & $<---$ & BPT PsyCon & .738 & .180 & 4.097 & $* * *$ \\
\hline Q7Q2 & $<---$ & BPT PsyCon & 1.000 & & & $* * *$ \\
\hline Q7Q4 & $<---$ & BPT PsyCon & 1.186 & .188 & 6.315 & $* * *$ \\
\hline \hline Standardized Regression
\end{tabular}

Standardized Regression

\begin{tabular}{lccc}
\hline & & & Estimate \\
\hline BPT_PsyCon & $<--$ & Work_PsyCon & .842 \\
\hline Q5S2 & $<--$ & Work_PsyCon & .882 \\
\hline Q5S6 & $<--$ & Work_PsyCon & .716 \\
\hline Q5S5 & $<--$ & Work_PsyCon & .578 \\
\hline BPT_Effect & $<---$ & BPT PsyCon & .652 \\
\hline Q7Q2 & $<---$ & BPT PsyCon & .855 \\
\hline Q7Q4 & $<---$ & BPT PsyCon & .911 \\
\hline \hline
\end{tabular}

To evaluate the fitness of our research model, we used the indexes such as, the normed-fit-index (NFI), the relative-fit-index (RFI), and the respective root mean square of approximation (RMSEA). The recommended fit values for NFI and RFI are upper than 0.90 [19], and a lower RMSEA indicates a better model with the suggested maximum being 0.08 [20] or 0.06 [21]. Based on these indexes, our research result can be regarded as acceptable.

\section{CONCLUSION}

The purpose of this paper was to examine the correlation between psychological contracts and BPT effect which should be one of the success factors of BPT focusing on trust in BPT. There was a positive relationship between the latent variable, work psychological contracts and the BPT psychological contracts, and the latent variable, BPT psychological contracts has a positive impact on the observed variable, BPT effect. However, there was no significant relationship between work psychological contracts and BPT effect. This means the methodology to 
make BPT psychological contracts is significant to accomplish BPTs effectively. This means methodologies to establish BPT psychological contracts is significant to accomplish BPT effectively. Possible options are such as sending messages that the company is seriously and vigorously making efforts to accomplish BPT, providing supporting arrangements and necessary resources for BPT, and involving the CEO, the equivalents, and champions/elites in the project.

An illustrative example of establishing BPT psychological contracts is Cross-Functional Teams (CFT). The purpose of CFT is to plan, implement, and promote/enforce necessary cooperate-wide large-scale BPT that go beyond individual functional departments. More specifically, rules such as the followings are implemented so that the team members feel secured and concentrate on the project easily; the RPT project is prioritized to ordinary business routines and the most suitable members from each functional department are assigned to the project; various and affluent supports for managing projects such as project management guidelines and advisors/consultants are provided; evaluation procedures of the project outcome is clearly specified and it is evaluated fairly; the BPT proposal is promoted and enforced by the CEO or the equivalents when once it is officially accepted so that political sabotages are eliminated. Active involvement of the CEO or the equivalents in respective steps is seen, which becomes a clear message to convey that the company is serious about the accomplishment of the BPT.

For our future studies, multiple case studies focusing on the detailed processes of establishing BPT psychological contracts including above issues will be examined.

\section{ACKNOWLEDGEMENT}

This work was supported in part by a Grant-in-Aid for Scientific Research (16K03819). Also, we really appreciate the firms who cooperated in the questionnaire.

\section{REFERENCES}

[1] Organizsation for Economic Co-operation and Development (OECD). OECD Compendium of Productivity Indicators. (2016). [Online].

Available: https://www.oecd-ilibrary.org/industry-and-services/oecd-compendi um-of-productivity-indicators-2016_pdtvy-2016-en

[2] M. Hojo, "Recent issues in the Japanese labor market," Journal of International Studies, School of International Studies, Kwansei Gakuin University, vol. 6, no. 3, pp. 63-73, 2017.

[3] Japan Productivity Center (JPC), International Comparison of Labor Productivity 2016. [Online]. Available: https://www.jpc-net.jp/intl_comparison/intl_comparison_2016R2. Pdf

[4] T. Higano, "IT ni Yoru work style Henkaku," Nomura Research Institute, pp. 16-19, 2009.

[5] A. Khosravi, "Business process rearrangement and renaming: A new approach to process orientation and improvement," Business Process Management Journal, vol. 22, no. 1, pp.116-139, 2005.

[6] D. M. Rousseau and J. M. Parks, "Psychological and implied contracts in organizations," Employee Responsibilities and Rights Journal, vol. 2, no. 2, pp. 121-139, 1989.

[7] D. M. Rousseau, Psychological Contracts in Organizations: Understanding Written and Unwritten Agreements, Thousand Oaks, CA: Sage. 1995.

[8] L. M. Shore and L. Tetrick, "The psychological contract as an explanatory framework in the employment relationship," Trends in Organizational Behavior, vol. 1, pp. 91-109, 1994.

[9] R. O. A. Lopes, R. Roberto, and E. L. Qualharini, "The psychological contract and project management as a core competence of the organization," Procedia - Social and Behavioral Sciences, vol. 226, pp. 148-155, July 2016.

[10] M. Hammer and J. Champy, "Re-engineering the corporation: A manifesto for business revolution," Harper Business, 1993.

[11] V. Grover, S. R. Jeong, W. J. Kettinger, and J. T. C. Teng, "The implementation of business process re-engineering," Journal of Management Information Systems, vol. 12, no. 1. pp. 109-144, 1995.

[12] M. J. Earl, J. L. Sampler, and J. E. Short, "Strategies for business process re-engineering: evidence from field studies," Journal of Management Information Systems, vol. 12, no. 1, pp. 31-56, Summer 1995.

[13] M. Attaran, "Exploring the relationship between information technology and business process re-engineering," Information and Management, vol. 41, no. 5, pp.585-596, May 2004.

[14] M. Larsen and M. Myers, "BPR success or failure? A business process reengineering project in the financial services industry," in Proc. International Conference on Information Systems (ICIS) 1997, pp. 367-382, 1997.

[15] M. Al-Mashari and M. Zairi, "BPR implementation process: An analysis of key success and failure factors," Business Process Management Journal, vol. 5, no. 1, pp. 87-112, 1999.

[16] Y. Takei, I. Okuda, and K. Iizuka, "CSF of ERP system implementation: Findings from literature survey," SIG Technical Reports, pp. 1-6, 2013.

[17] R. Suzuki, "Soshiki to Kojin: Career no Hattatsu to soshiki no commitment no Henka," Hakuto Shobo, 2002.

[18] Y. Hattori, "Psychological contract in Japanese companies: Invisible contracts between employees and emplyer," Hakuto Shobo, 2011.

[19] J. F. H. Hair, R. E. Anderson, R. L. Tatham, and W. C. Black, Multivariate Data Analysis, (5th Ed.). Engelwood Cliffs, NJ Prentice Hall. 1998.

[20] R. C. M. Callum, M. W. Browne, and H. Sugawara, "Power analysis and determination of sample size for covariance structure modeling," Psychological Methods, vol. 1, no. 2, pp.130-49, 1996.

[21] L. T. Hu and P. M. Bentler, "Cutoff criteria for fit indexes in covariance structure analysis: Conventional criteria versus new alternatives," Structural Equation Modeling, vol. 6, pp. 1-55.1999.

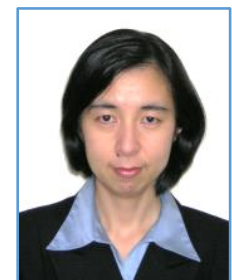

Kayo Iizuka has been engaged in the field of business and information system. After she had joined Fujitsu Ltd, Price Waterhouse Consultants Co. Ltd. (Japan) and Nomura Research Institute. She is currently a professor at the School of Network and Information, Senshu University, Japan. She was a visiting researcher at Kyoto University (2014-2015). She got her Ph.D. degree (in systems management) from Tsukuba University, and her M.B.A. degree from Keio University.

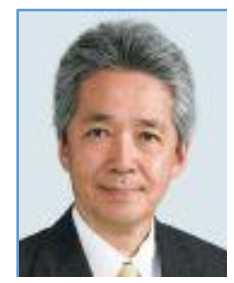

Chihiro Suematsu is a professor at the Graduate School of Management / Economics, Kyoto University. He has been engaged in the field of business management, from organization modeling to information system management. He worked for JGC Corporation, McKinsey \& Company, Japan, and Keio University. He received his Ph.D. degree (in Economics) from Kyoto University, and M.B.A. degree from Stanford University. 